\title{
Discrete similariton regime for energy scaling of modelocked thin-disk lasers
}

\author{
F. Ö. Ilday ${ }^{1,2,3}$, M. Hoffmann ${ }^{4}$, and C. J. Saraceno ${ }^{4}$ \\ 1- Department, of Physics, Bilkent University, Ankara 06800, Turkey \\ 2 - Department of Electrical and Electronics Engineering, Bilkent University, Ankara 06800, Turkey \\ 3- UNAM - National Nanotechnology Research Center and Institute of Materials Science and Nanotechnology, Bilkent University, Ankara \\ 06800, Turkey \\ 3- Ruhr Universität Bochum, Universitätsstraße 150, 44801, Bochum, Germany \\ Authore-mail address: clara.saraceno@ruhr-uni-bochum.de
}

\begin{abstract}
We investigate energy scaling of modelocked thin-disk lasers via modelocking regimes which are more tolerant in terms of maximum nonlinearity than soliton modelocking. Our numerical results show promise for mJ-level pulse energies without requiring vacuum.๑ 2018 The Author(s) OCIS codes: (140.0140) Lasers and Optics; (140.4050) Mode-locked lasers; (320.7090) Ultrafast Lasers
\end{abstract}

\section{Introduction}

Since their first demonstration, modelocked thin-disk lasers (TDLs) have consistently surpassed other modelocked oscillator technologies in terms of achievable pulse energy (up to $80 \mu \mathrm{J}$ [1]) and average power (up to $275 \mathrm{~W}$ [2]) by several orders of magnitude, reaching comparable levels to that of state-of-the-art amplifiers at MHz repetition rate.

However, several crucial challenges need to be overcome to take this technology to the next level. One critical challenge is achieving stable pulse formation at extremely high intracavity pulse energies of hundreds of microujoules to millijoules. Surprisingly, state-of-the-art results in this area have so far only been achieved in modelocking regimes where soliton pulse shaping is dominant (i.e. soliton modelocking with semiconductor saturable absorber mirrors or Kerr lens modelocking), in which only small nonlinear phase shifts are tolerable, ultimately limiting pulse energy scaling. So far, mitigating strategies to avoid these limitations have mostly targeted a reduction of intracavity nonlinearity, for example by operating in vacuum to minimize the nonlinearity of air [1] [2], or by decreasing intracavity pulse energy (without sacrificing output) via an increase of the gain per roundtrip [3]. Operation in the positive dispersion regime has been demonstrated both with SESAM-modelocked [4] and KLM TDLs [5] however in regimes of small GDD, with only a small advantage in terms of tolerable nonlinearity (and thus a very small advantage compared to soliton modelocking). The exploration of modelocking mechanisms in the normal dispersion regime which are more tolerant to large nonlinearities, thus more appropriate for millijoule-class pulse formation, remained so far widely unexplored, because the operation parameters of TDLs (in particular the small gain per roundtrip) appear at first glance unsuitable to support them.

Here, we explore the possibility of applying the similariton [6][7] modelocking regime for energy scaling of highenergy modelocked TDLs. This regime is commonly applied in modelocked fiber lasers, and has successfully demonstrated its energy scaling potential for this technology. We show that these advantages can potentially be extended to the case of modelocked TDLs, where the average power and pulse energy reachable is significantly larger. In this goal, we propose an active multipass scheme with overall large positive dispersion (introduced in discrete steps), gain and continuous nonlinearity to enable this modelocking scheme in this laser geometry. We refer to this regime the discrete similariton regime because gain, dispersion and much of nonlinearity do not occur simultaneously, but in discrete steps in the thin disk, air in between and dispersive mirrors of multipass cell, respectively. Yet, the discrete similariton retains the essential nonlinearity-resistant characteristic of a regular similariton. Our numerical investigation indicate that this is a promising path to increase the pulse energy achievable directly from modelocked oscillators towards the millijoule level, while additionally simplifying their implementation by eliminating the need for operation in cumbersome vacuum chambers.

\section{Proposed geometry and simulation results}

A schematic of the proposed layout, which was investigated numerically is presented in Fig. 1. The use of an active multi-pass cell (AMC) [3] with positive dispersive mirrors (DMs) and multiple gain bounces per resonator roundtrip can be designed to support self-similar pulse evolution. Large and continuous nonlinearity is achieved via propagation of the energetic pulses in air of the long multi-pass cell, and discrete (small) nonlinearity is achieved at every bounce on the disk: this contribution is non-negligible for very large number of disk passes. A section outside of the AMC with negative dispersion is required to support similariton modelocking. The overall cavity operates with small net positive dispersion, and large gain. Alternatively, similar concept can be applied in the dissipative soliton regime, with smaller gain and large positive dispersion, introduced in a Herriott-type multi-pass cell. 
discrete similariton TDL

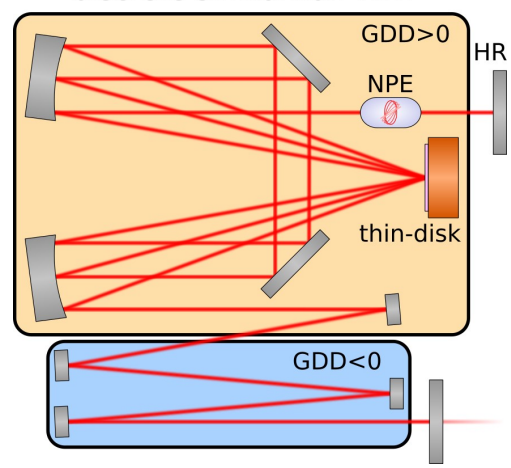

Intracavity pulse energy: $1.25 \mathrm{~mJ}$

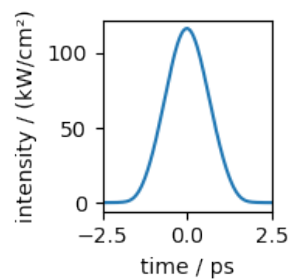

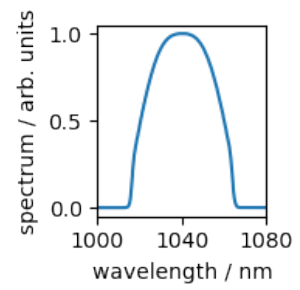

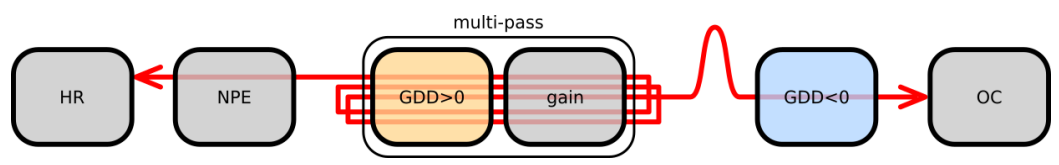

Fig.1 - (left) schematic of the concept of a discrete similariton TDL, illustrated with only 3-passes (right) Results of our simulations with a 10pass AMC (corresponding to 20 passes over the gain disk and mirrors) with total positive GDD of $+40 \mathrm{kfs}^{2}$ ( $+80 \mathrm{kfs} 2$ per roundtrip) of positive dispersion distributed over 20 bounces inside the AMC, and negative dispersion introduced outside of the AMC of $-35 \mathrm{kfs}{ }^{2}$.

We model pulse propagation using a generalized complex Ginzburg-Landau equation that accounts for Kerr nonlinearity, Raman scattering, dispersion and where appropriate gain, loss and spectral filtering [7]. The gain model includes the effects of finite bandwidth (here $40 \mathrm{~nm}$, centered at $1040 \mathrm{~nm}$, as a generic Yb-doped gain medium) and gain saturation. The laser cavity includes the active MPC, where the mirrors provide positive dispersion (total $+40000 \mathrm{fs}^{2}$ ) and air. The thin disk is modeled with a small signal gain coefficient of $0.5 \mathrm{~dB}$ per pass, and the total nonlinearity of the cavity is set to correspond to recent experimental results on high-energy TDLs [1] $(\gamma=20 \mathrm{mrad} / \mathrm{MW})$. The AMC simulated contained 10 propagation passes (20 per roundtrip), thus a total small signal gain of $10 \mathrm{~dB}$. We included another set of DMs providing negative dispersion ( $\left.-35000 \mathrm{fs}^{2}\right)$. We also add the response of a saturable absorber, in this case modeled as a nonlinear-polarization evolution NPE-saturable absorber with $50 \%$ modulation depth. Finally, we introduce a reflective output coupler mirror with $10 \%$ linear loss, which also acts as a narrow ( $\sim 30 \mathrm{~nm}$-wide) filter. The resonator was $3 \mathrm{MHz}$ long.

Fig.1 (right) shows a configuration where we obtain intracavity pulse energies of $1.25 \mathrm{~mJ}$, and output pulses with 1.5 ps duration, with predominantly linear chirp. This energy is nearly an order of magnitude higher to what is typically achieved intracavity with soliton modelocked TDLs operated in vacuum [1] - but in this case in air. This shows the key advantage of this regime: the longer average duration of the pulse in the resonator, with smaller peak power, and additionally an order of magnitude higher tolerable nonlinear phase shifts (typically several $\pi \mathrm{rad}$ ) allows to achieve much higher pulse energy. In this specific case, our simulation predicts $100 \mu \mathrm{J}$ output pulse energy, however, given the high gain in this configuration, output energies as high as $500 \mu \mathrm{J}$, at $3 \mathrm{MHz}$ repetition rate are expected to be possible to extract from the resonator, depending on the specific implementation of other components. The dechirped pulse duration, in this case, would allow to reach $100 \mathrm{fs}$, however, this will most likely depend on the chosen $\mathrm{Yb}$-doped gain material. We note that it is not uncommon in this regime to exceed the gain bandwidth of the gain material, which should also relax gain material constraints. In these first simulations, we used a large modulation depth to relax other parameters, and focus on the potential of this technique for energy scaling. First simulations with low-modulation depth SESAMs also appear to be possible with comparable pulse energy levels. Additionally, we believe that at this high pulse energy level, NPE can be considered in the case of TDLs, which is also an interesting unexplored direction. In the next steps, we target to further explore this regime to achieve higher output energy levels, as well as implement this concept in a proof-of-principle experimental setup.

[1] C. J. Saraceno, F. Emaury, C. Schriber, M. Hoffmann, M. Golling, T. S ,dmeyer, et al., "Ultrafast thin-disk laser with $80 \mu \mathrm{J}$ pulse energy and 242 W of average power," Optics Letters, vol. 39, pp. 9-12, 2014/01/01 2014.

[2] C. J. Saraceno, F. Emaury, O. H. Heckl, C. R. E. Baer, M. Hoffmann, C. Schriber, et al., "275 W average output power from a femtosecond thin disk oscillator operated in a vacuum environment," Opt. Express, vol. 20, pp. 23535-23541, 2012

[3] D. Bauer, I. Zawischa, D. H. Sutter, A. Killi, and T. Dekorsy, "Mode-locked Yb:YAG thin-disk oscillator with $41 \mu \mathrm{J}$ pulse energy at $145 \mathrm{~W}$ average infrared power and high power frequency conversion," Opt. Express, vol. 20, pp. 9698-9704, 2012.

[4] G. Palmer, M. Schultze, M. Siegel, M. Emons, U. Bünting, and U. Morgner, "Passively mode-locked $\left.\mathrm{Yb}: \mathrm{KLu}_{(\mathrm{WO}}\right)_{2}$ thin-disk oscillator operated in the positive and negative dispersion regime," Opt. Lett., vol. 33, pp. 1608-1610, 2008.

[5] O. Pronin, J. Brons, C. Grasse, V. Pervak, G. Boehm, M. C. Amann, et al., "High-power Kerr-lens mode-locked Yb:YAG thin-disk oscillator in the positive dispersion regime," Optics Letters, vol. 37, pp. 3543-3545, Sep 12012.

[6] F. O. Ilday, J. R. Buckley, W. G. Clark, and F. W. Wise, "Self-similar evolution of parabolic pulses in a laser," Physical Review Letters, vol. 92, May 282004.

[7] B. Oktem, C. Ulgudur, and F. O. Ilday, "Soliton-similariton fibre laser," Nature Photonics, vol. 4, pp. 307-311, May 2010. 